\title{
Arbitrary Order Fractional Difference Operators with Discrete Exponential Kernels and Applications
}

\author{
Thabet Abdeljawad, 1 Qasem M. Al-Mdallal, ${ }^{2}$ and Mohamed A. Hajji ${ }^{2}$ \\ ${ }^{1}$ Department of Mathematics and Physical Sciences, Prince Sultan University, P.O. Box 66833, Riyadh 11586, Saudi Arabia \\ ${ }^{2}$ Department of Mathematical Sciences, United Arab Emirates University, P.O. Box 17551, Al Ain, Abu Dhabi, UAE \\ Correspondence should be addressed to Thabet Abdeljawad; tabdeljawad@psu.edu.sa
}

Received 3 April 2017; Accepted 25 May 2017; Published 21 June 2017

Academic Editor: Garyfalos Papashinopoulos

Copyright (C) 2017 Thabet Abdeljawad et al. This is an open access article distributed under the Creative Commons Attribution License, which permits unrestricted use, distribution, and reproduction in any medium, provided the original work is properly cited.

Recently, Abdeljawad and Baleanu have formulated and studied the discrete versions of the fractional operators of order $0<\alpha \leq 1$ with exponential kernels initiated by Caputo-Fabrizio. In this paper, we extend the order of such fractional difference operators to arbitrary positive order. The extension is given to both left and right fractional differences and sums. Then, existence and uniqueness theorems for the Caputo (CFC) and Riemann (CFR) type initial difference value problems by using Banach contraction theorem are proved. Finally, a Lyapunov type inequality for the Riemann type fractional difference boundary value problems of order $2<\alpha \leq 3$ is proved and the ordinary difference Lyapunov inequality then follows as $\alpha$ tends to 2 from right. Illustrative examples are discussed and an application about Sturm-Liouville eigenvalue problem in the sense of this new fractional difference calculus is given.

\section{Introduction}

In the last few decades, the continuous and discrete fractional differential equations have received considerable interest due to their importance in many scientific fields; see, by way of example not exhaustive enumeration, [1-7].

In [8], the authors introduced a fractional derivative with an exponential kernel which tends to the ordinary derivative as $\alpha$ tends to 1 . More properties of this fractional derivative have been studied in [9], where the correspondent fractional integral operator was formulated. Then, the authors in [7] defined the left and right fractional derivatives with exponential kernel in the Riemann sense and formulated the right fractional derivatives in the sense of Caputo-Fabrizio with complete investigation to the correspondent fractional integrals and all the discrete versions with integration and summation by parts applied in the fractional and discrete fractional variational calculus. Then, very recently, the same authors proved an interesting monotonicity result in the sense of this new fractional difference calculus in [10].

In the same direction, for the purpose of providing more fractional derivatives with different nonsingular kernels, the authors in [11] defined a fractional operator with
Mittag-Leffler kernel and in $[12,13]$ the complete details and discrete versions have been studied. The exponential kernel fractional derivatives and hence their discrete counterparts are quite different from the Mittag-Leffler kernel fractional operators. For example, the integral operator corresponding to exponential kernel fractional derivatives consists of a multiple of the function $f$ added to a multiple of the integration of $f$, whereas the Mittag-Leffler kernel correspondent integral operator consists of a multiple of $f$ and a Riemann-Liouville fractional integral of the same order. Also, the monotonicity coefficient of the CFR fractional difference operator of order $0<\alpha \leq 1$ is $\alpha$ as shown in [10], whereas for the discrete Mittag-Leffler CFR operator is $\alpha^{2}$ as proven in [14].

Motivated, by what we mentioned above, we extend the order of fractional difference type operators with discrete exponential kernels to arbitrary positive order, prove existence and uniqueness theorems for the fractional initial value difference problems, and finally prove a Lyapunov type inequality for the CFR fractional difference operators of order $2<\alpha \leq 3$. The ordinary discrete Lyapunov inequality is then confirmed as $\alpha$ tends to 2 from the right not as in the case of the classical fractional difference as $\alpha$ tends to 2 from the left [15]. For various fractional Lyapunov extensions we refer, for 
example, to [16-29]. All the authors there were motivated by the following theorem on ordinary Lyapunov inequality.

Theorem 1 (see [30]). If the boundary value problem

$$
y^{\prime \prime}(t)+q(t) y(t)=0, \quad t \in(a, b), \quad y(a)=y(b)=0,
$$

has a nontrivial solution, where $q$ is a real continuous function; then

$$
\int_{a}^{b}|q(s)| d s>\frac{4}{b-a} .
$$

Notice that inequality (2) is known as the classical Lyapunov inequality. It is worth mentioning that Cheng [31] had pointed out that Lyapunov neither stated nor proved Theorem 1 but he only stated the following result.

Theorem 2 (see [26]). Let $q(t)$ be a nontrivial, continuous, and nonnegative function with period $\omega$ and let

$$
\int_{0}^{\omega} q(s) d s \leq \frac{4}{\omega} .
$$

Then the roots of the characteristic equation corresponding to Hills equation

$$
x^{\prime \prime}(t)+q(t) x(t)=0, \quad-\infty<t<\infty,
$$

are purely imaginary with modulus one.

For the classical fractional calculus which is behind many extensions, we refer the reader to [32-35] and for the sake of comparison with the classical discrete fractional case we refer to [36] and the references therein. In addition, for the discrete fractional operators and their duality we refer to [37-39].

The article will be organised as follows: In the remaining part of this section we shall give some basics about the discrete CFC and CFR fractional differences and their correspondent sums as used in $[7,10]$. In Section 2, we extend the order of CFC and CFR fractional differences and their correspondent sums to arbitrary positive order and give some illustrative examples. In Section 3, we prove some existence and uniqueness theorems by means of Banach fixed point theorem and give some illustrative examples. In Section 4, we prove a Lyapunov type inequality for a fractional CFR difference boundary value problem of order $2<\alpha \leq 3$ and give an application to the fractional difference Sturm-Liouville Eigenvalue problem (SLEP) to enrich the applicability of our proven Lyapunov inequality in the frame of fractional difference operators with discrete exponential kernels.

\section{Preliminaries}

Definition 3 (see [36]). For $\alpha>0, a \in \mathbb{R}, \rho(s)=s-1$, and $f$ a real-valued function defined on $\mathbb{N}_{a}=\{a, a+1, \ldots\}$, the left Riemann-Liouville fractional sum of order $\alpha>0$ is defined by

$$
\left({ }_{a} \nabla^{-\alpha} f\right)(t)=\frac{1}{\Gamma(\alpha)} \sum_{s=a+1}^{t}(t-\rho(s))^{\overline{\alpha-1}} f(s)
$$

This is fractionalising of the $n$-iterated nabla sum

$$
\left({ }_{a} \nabla^{-n} f\right)(t)=\frac{1}{(n-1) !} \sum_{a+1}^{t}(t-\rho(s))^{\overline{n-1}} f(s)
$$

The right fractional integral ending at $b$, where usually we assume that $a \equiv b(\bmod 1)$, is defined by

$$
\left(\nabla_{b}^{-\alpha} f\right)(t)=\frac{1}{\Gamma(\alpha)} \sum_{s=t}^{b-1}(s-\rho(t))^{\overline{\alpha-1}} f(s),
$$

where $t^{\bar{\alpha}}=\Gamma(t+\alpha) / \Gamma(t)$ and $\Gamma(t)$ is the well-known gamma special function of $t$.

Definition 4 (see $[7,10]$ ). For $\alpha \in(0,1)$ and $f$ defined on $\mathbb{N}_{a}$, or ${ }_{b} \mathbb{N}=\{b, b-1, \ldots\}$ in right case, we have the following definitions:

(i) The left (nabla) CFC fractional difference is given by

$$
\begin{aligned}
\left({ }_{a}^{\mathrm{CFC}} \nabla^{\alpha} f\right)(t) & =\frac{B(\alpha)}{1-\alpha} \sum_{s=a+1}^{t}\left(\nabla_{s} f\right)(s)(1-\alpha)^{t-\rho(s)} \\
& =B(\alpha) \sum_{s=a+1}^{t}\left(\nabla_{s} f\right)(s)(1-\alpha)^{t-s}
\end{aligned}
$$

(ii) The right (nabla) CFC fractional difference has the following form:

$$
\begin{aligned}
\left({ }^{\mathrm{CFC}} \nabla_{b}^{\alpha} f\right)(t) & =\frac{B(\alpha)}{1-\alpha} \sum_{s=t}^{b-1}\left(-\Delta_{s} f\right)(s)(1-\alpha)^{s-\rho(t)} \\
& =B(\alpha) \sum_{s=t}^{b-1}\left(-\Delta_{s} f\right)(s)(1-\alpha)^{s-t}
\end{aligned}
$$

(iii) The left (nabla) CFR fractional difference is written as

$$
\begin{aligned}
\left({ }_{a}^{\mathrm{CFR}} \nabla^{\alpha} f\right)(t) & =\frac{B(\alpha)}{1-\alpha} \nabla_{t} \sum_{s=a+1}^{t} f(s)(1-\alpha)^{t-\rho(s)} \\
& =B(\alpha) \nabla_{t} \sum_{s=a+1}^{t} f(s)(1-\alpha)^{t-s} .
\end{aligned}
$$
by

(iv) The right (nabla) CFR fractional difference is given

$$
\begin{aligned}
\left({ }^{\mathrm{CFR}} \nabla_{b}^{\alpha} f\right)(t) & =\frac{B(\alpha)}{1-\alpha}\left(-\Delta_{t}\right) \sum_{s=t}^{b-1} f(s)(1-\alpha)^{s-\rho(t)} \\
& =B(\alpha)\left(-\Delta_{t}\right) \sum_{s=t}^{b-1} f(s)(1-\alpha)^{s-t}
\end{aligned}
$$

where $B(\alpha)$ is a normalization positive constant depending on $\alpha$ satisfying $B(0)=B(1)=1,(\nabla g)(t)=g(t)-g(t-1)$, and $(\Delta g)(t)=g(t+1)-g(t)$. 
In [7, 8], it was verified that $\left({ }_{a}^{\mathrm{CF}} \nabla^{-\alpha}{ }_{a} \nabla^{\alpha} f\right)(t)=f(t)$ and $\left({ }_{a}^{\mathrm{CFR}} \nabla^{\alpha}{ }_{a} \nabla^{-\alpha} f\right)(t)=f(t)$. Also, in the right case $\left({ }^{\mathrm{CF}} I_{b}^{\alpha}{ }^{\alpha F R} \nabla_{b}^{\alpha} f\right)(t)=f(t)$ and $\left({ }^{\mathrm{CFR}} \nabla_{b}^{\alpha}{ }^{\mathrm{CF}} \nabla_{b}^{-\alpha} f\right)(t)=f(t)$. From $[7,8]$ we recall the relation between the CFC and CFR fractional differences as

$$
\begin{aligned}
\left({ }_{a}^{\mathrm{CFC}} \nabla^{\alpha} f\right)(t)= & \left({ }_{a}^{\mathrm{CFR}} \nabla^{\alpha} f\right)(t) \\
& -\frac{B(\alpha)}{1-\alpha} f(a)(1-\alpha)^{t-a},
\end{aligned}
$$

and for the right case by

$$
\begin{aligned}
\left({ }^{\mathrm{CFC}} \nabla_{b}^{\alpha} f\right)(t)= & \left({ }^{\mathrm{CFR}} \nabla_{b}^{\alpha} f\right)(t) \\
& -\frac{B(\alpha)}{1-\alpha} f(b)(1-\alpha)^{b-t} .
\end{aligned}
$$

Notice that we extend Definition 4 to arbitrary $\alpha>0$ in the next section.

Lemma 5 (see [7]). For $0<\alpha<1$, we have

$$
\begin{aligned}
& \left({ }_{a}^{C F} \nabla_{a}^{-\alpha} C F C \nabla^{\alpha} f\right)(t)=f(t)-f(a), \\
& \left({ }^{C F} \nabla_{b}^{-\alpha} C F C \nabla_{b}^{\alpha} f\right)(t)=f(t)-f(b) .
\end{aligned}
$$

Notation. For a positive integer $n$, we have

(i) $\left(\nabla^{n} f\right)(t)=(\underbrace{\nabla \nabla \cdots \nabla}_{n \text { times }} f)(t)$.

(ii) $\left(\Delta^{n} f\right)(t)=(\underbrace{\Delta \Delta \cdots \Delta f}_{n \text { times }})(t)$.

(iii) $\left({ }_{\ominus} \Delta^{n} f\right)(t)=(-1)^{n}\left(\Delta^{n} f\right)(t)$.

\section{Higher Order Fractional Differences and Sums}

Definition 6. Let $n<\alpha \leq n+1$ and $f$ be defined on $\mathbb{N}_{a} \cap{ }_{b} \mathbb{N}$. Set $\beta=\alpha-n$ and define

$$
\begin{aligned}
& \left({ }_{a}^{\mathrm{CFC}} \nabla^{\alpha} f\right)(t)=\left({ }_{a}^{\mathrm{CFC}} \nabla^{\beta} \nabla^{n} f\right)(t), \\
& \left({ }_{a}^{\mathrm{CFR}} \nabla^{\alpha} f\right)(t)=\left({ }_{a}^{\mathrm{CFR}} \nabla^{\beta} \nabla^{n} f\right)(t) .
\end{aligned}
$$

The associated fractional sum is given by

$$
\left({ }_{a}^{\mathrm{CF}} \nabla^{-\alpha} f\right)(t)=\left({ }_{a} \nabla_{a}^{-n}{ }_{a}^{\mathrm{CF}} \nabla^{-\beta} f\right)(t) .
$$

Note that if we use the convention that $\left({ }_{a} \nabla^{-0} f\right)(t)=f(t)$, then for the case $0<\alpha \leq 1$ we have $\beta=\alpha$ and hence we obtain Definition 4. Also, the convention $\left(\nabla^{0} f\right)(t)=f(t)$ leads to $\left({ }_{a}^{\mathrm{CFR}} \nabla^{\alpha} f\right)(t)$ and $\left({ }_{a}^{\mathrm{CFC}} \nabla^{\alpha} f\right)(t)$ as in Definition 4 for $0<\alpha \leq 1$.

Remark 7. In Definition 6, if we let $\alpha=n+1$ then $\beta=1$ and hence $\left({ }_{a}^{\mathrm{CFR}} \nabla^{\alpha} f\right)(t)=\left({ }_{a}^{\mathrm{CFR}} \nabla^{1} \nabla^{n} f\right)(t)=\left(\nabla^{n+1} f\right)(t)$. Also, by noting that $\left({ }_{a}^{\mathrm{CF}} \nabla^{-1} f\right)(t)=\left({ }_{a} \nabla^{-1} f\right)(t)$, we see that for $\alpha=n+1$ we have $\left({ }_{a}^{\mathrm{CF}} \nabla^{-\alpha} f\right)(t)=\left({ }_{a} \nabla^{-(n+1)} f\right)(t)$. Also, for $0<\alpha \leq 1$ we reobtain the concepts defined in Definition 4 . Therefore, our generalization to higher order case is confirmed.

Analogously, in the right case we have the following extension.

Definition 8. Let $n<\alpha \leq n+1$ and $f$ be defined on $\mathbb{N}_{a} \cap{ }_{b} \mathbb{N}$. Set $\beta=\alpha-n$. Then $\beta \in(0,1]$ and we define

$$
\begin{aligned}
& \left({ }^{\mathrm{CFC}} \nabla_{b}^{\alpha} f\right)(t)={ }^{\mathrm{CFC}} \nabla_{b}^{\beta}\left({ }_{\ominus} \Delta^{n} f\right)(t), \\
& \left({ }^{\mathrm{CFR}} \nabla_{b}^{\alpha} f\right)(t)={ }^{\mathrm{CFR}} \nabla_{b}^{\beta}\left({ }_{\ominus} \Delta^{n} f\right)(t) .
\end{aligned}
$$

The associated fractional integral is given by

$$
\left({ }^{\mathrm{CF}} \nabla_{b}^{-\alpha} f\right)(t)=\left(\nabla_{b}^{-n}{ }^{\mathrm{CF}} \nabla_{b}^{-\beta} f\right)(t) .
$$

An immediate extension of (12) and (13) by using Definition 6 is the following.

Proposition 9. For $f$ defined on $\mathbb{N}_{a} \cap_{b} \mathbb{N}$ and $n<\alpha \leq n+1$, we have

$$
\begin{aligned}
\left({ }_{a}^{C F C} \nabla^{\alpha} f\right)(t)= & \left({ }_{a}^{C F R} \nabla^{\alpha} f\right)(t) \\
& -\frac{B(\alpha)}{1-\alpha}\left(\nabla^{n} f\right)(a)(1-\alpha)^{t-a},
\end{aligned}
$$

and for the right case

$$
\begin{aligned}
\left({ }^{C F C} \nabla_{b}^{\alpha} f\right)(t)= & \left({ }^{C F R} \nabla_{b}^{\alpha} f\right)(t) \\
& -\frac{B(\alpha)}{1-\alpha}\left({ }_{\ominus} \Delta^{n} f\right)(b)(1-\alpha)^{b-t} .
\end{aligned}
$$

Next proposition explains the action of the arbitrary order sum operator ${ }_{a}^{\mathrm{CF}} \nabla^{-\alpha}$ on the arbitrary order CFR and CFC differences (and vice versa) and the action of the CFR difference on the CF correspondent sum operator.

Proposition 10. For $u(t)$ defined on $\mathbb{N}_{a} \cap_{b} \mathbb{N}$ and for some $n \in$ $\mathbb{N}_{0}$ with $n<\alpha \leq n+1$, we have

(i) $\left({ }_{a}^{C F R} \nabla^{\alpha}{ }_{a}^{C F} \nabla^{-\alpha} u\right)(t)=u(t)$.

(ii) $\left({ }_{a}^{C F} \nabla^{-\alpha}{ }_{a} \nabla^{\alpha} u\right)(t)=u(t)-\sum_{k=0}^{n-1}\left(\left(\nabla^{k} u\right)(a) / k !\right)(t-a)^{\bar{k}}$.

(iii) $\left({ }_{a}^{C F} \nabla^{-\alpha} C F C{ }_{a}^{\alpha} u\right)(t)=u(t)-\sum_{k=0}^{n}\left(\left(\nabla^{k} u\right)(a) / k !\right)(t-a)^{\bar{k}}$.

Proof. (i) By Definition 6 and the statement after Definition 4, we have

$$
\begin{aligned}
\left({ }_{a}^{\mathrm{CFR}} \nabla^{\alpha}{ }_{a} \nabla^{-\alpha} u\right)(t) & ={ }_{a}^{\mathrm{CFR}} \nabla^{\beta} \nabla_{a}^{n} \nabla^{-n}{ }_{a}^{\mathrm{CF}} \nabla^{-\beta} u(t) \\
& ={ }_{a}^{\mathrm{CFR}} \nabla_{a}^{\beta}{ }_{a} \nabla^{-\beta} u(t)=u(t) .
\end{aligned}
$$


(ii) By Definition 6 and the statement after Definition 4, we have

$$
\begin{aligned}
\left({ }_{a}^{\mathrm{CF}} \nabla_{a}^{-\alpha} \nabla^{\mathrm{CFR}} u\right)(t) & =\left({ }_{a} \nabla_{a}^{-n}{ }_{a}^{\mathrm{CF}}{ }_{a}^{-\beta} \nabla^{\beta} \nabla^{n} u\right)(t) \\
& ={ }_{a} \nabla^{-n} \nabla^{n} u(t) \\
& =u(t)-\sum_{k=0}^{n-1} \frac{\left(\nabla^{k} u\right)(a)}{k !}(t-a)^{\bar{k}},
\end{aligned}
$$

where $\beta=\alpha-n$.

(iii) By Lemma 5 applied to $f(t)=\left(\nabla^{n} u\right)(t)$, we have

$$
\begin{aligned}
& \left({ }_{a}^{\mathrm{CF}} \nabla^{-\alpha}{ }_{a}^{\mathrm{CFC}} \nabla^{\alpha} u\right)(t)={ }_{a} \nabla^{-n}{ }_{a} \nabla^{-\beta C F C} \nabla^{\beta} \nabla^{n} u(t) \\
& ={ }_{a} \nabla^{-n}\left[\nabla^{n} u(t)-\left(\nabla^{n} u\right)(a)\right] \\
& =u(t)-\sum_{k=0}^{n-1} \frac{\left(\nabla^{k} u\right)(a)}{k !}(t-a)^{\bar{k}} \\
& -\left(\nabla^{n} u\right)(a) \frac{(t-a)^{\bar{n}}}{n !} \\
& =u(t)-\sum_{k=0}^{n} \frac{\left(\nabla^{k} u\right)(a)}{k !}(t-a)^{\bar{k}} \text {. }
\end{aligned}
$$

Using the facts that ${ }_{\ominus} \Delta^{n} \nabla_{b}^{-n} g(t)=g(t)$,

$$
\nabla_{b \ominus}^{n} \Delta^{n} g(t)=g(t)-\sum_{k=0}^{n-1} \frac{\left({ }_{\ominus} \Delta^{k} g\right)(b)}{k !}(b-t)^{\bar{k}},
$$

and making use of Lemma 5 and the statement after Definition 4 , we can state, for the right case, the following.

Proposition 11. For $u(t)$ defined on $\mathbb{N}_{a, b}$ and $\alpha \in(n, n+1]$, for some $n \in \mathbb{N}_{0}$, we have

(i) $\left({ }^{C F R} \nabla_{b}^{\alpha} C F \nabla_{b}^{-\alpha} u\right)(t)=u(t)$.

(ii) $\left({ }^{C F} \nabla_{b}^{-\alpha} C F R \nabla_{b}^{\alpha} u\right)(t)=u(t)-\sum_{k=0}^{n-1}\left(\left({ }_{\ominus} \Delta^{k} u\right)(b) / k !\right)(b-t)^{\bar{k}}$.

(iii) $\left({ }^{C F} \nabla_{b}^{-\alpha} C F C \nabla_{b}^{\alpha} u\right)(t)=u(t)-\sum_{k=0}^{n}\left(\left({ }_{\ominus} \Delta^{k} u\right)(b) / k !\right)(b-t)^{\bar{k}}$.

Example 12. Consider the initial value problem:

$$
\left({ }_{a}^{\mathrm{CFC}} \nabla^{\alpha} y\right)(t)=F(t)
$$

where $F(t)$ is defined on $\mathbb{N}_{a, b}=\mathbb{N}_{a} \cap{ }_{b} \mathbb{N}$. Let us consider two cases depending on the order $\alpha>0$ :

(i) Assume $0<\alpha \leq 1, y(a)=c$, and $F(a)=0$. By applying ${ }_{a}^{\mathrm{CF}} \nabla^{-\alpha}$ and making use of Proposition 10, we get the solution

$$
y(t)=c+\frac{1-\alpha}{B(\alpha)} F(t)+\frac{\alpha}{B(\alpha)} \sum_{s=a+1}^{t} F(s) .
$$

Notice that the condition $F(a)=0$ verifies the initial condition $y(a)=c$. In addition, when $\alpha \rightarrow 1$ we obtain the solution of the ordinary difference initial value problem $(\nabla y)(t)=F(t), y(a)=c$.

(ii) Assume $1<\alpha \leq 2, F(a)=0, y(a)=c_{1}$, and $(\nabla y)(a)=c_{2}$. By applying ${ }_{a}^{\mathrm{CF}} \nabla^{-\alpha}$ and making use of Proposition 10 and Definition 6 with $\beta=\alpha-1$, we obtain the solution

$$
\begin{gathered}
y(t)=c_{1}+c_{2}(t-a)+\frac{2-\alpha}{B(\alpha-1)} \sum_{s=a+1}^{t} K(s) \\
+\frac{\alpha-1}{B(\alpha-1)} \sum_{s=a+1}^{t}(t-\rho(s)) F(s) .
\end{gathered}
$$

Notice that the solution $y(t)$ verifies $y(a)=c_{1}$ without the use of $F(a)=0$. However, it verifies $(\nabla y)(a)=c_{2}$ under the assumption $F(a)=0$. Also, note that when $\alpha \rightarrow 2$ we recover the solution of the second-order ordinary difference initial value problem $\left(\nabla^{2} y\right)(t)=$ $F(t), y(a)=c_{1}$, and $(\nabla y)(a)=c_{2}$.

In the next section, we prove existence and uniqueness theorems for some types of CFC and CFR initial value difference problems.

Example 13. Consider the CFC difference boundary value problem

$$
\begin{aligned}
& \left({ }_{a}^{\mathrm{CFC}} \nabla^{\alpha} y\right)(t)+q(t) y(t)=0, \\
& 1<\alpha \leq 2, t \in \mathbb{N}_{a, b}, \quad y(a)=y(b)=0 .
\end{aligned}
$$

Then $\beta=\alpha-1$ and by Proposition 10 if we apply the operator ${ }_{a}^{\mathrm{CF}} \nabla^{-\alpha}$, we obtain the solution

$$
y(t)=c_{1}+c_{2}(t-a)-\left({ }_{a}^{\mathrm{CF}} \nabla^{-\alpha} q(\cdot) y(\cdot)\right)(t) .
$$

But

$$
\begin{aligned}
\left({ }_{a}^{\mathrm{CF}} \nabla^{-\alpha} q(\cdot) y(\cdot)\right)(t)= & \frac{1-\beta}{B(\beta)} \sum_{s=a+1}^{t} q(s) y(s) \\
& +\frac{\beta}{B(\beta)}{ }^{a} \nabla^{-2} q(t) y(t) .
\end{aligned}
$$

Hence, the solution has the form

$$
\begin{aligned}
y(t)= & c_{1}+c_{2}(t-a)-\frac{2-\alpha}{B(\alpha-1)} \sum_{s=a+1}^{t} q(s) y(s) \\
& -\frac{\alpha-1}{B(\alpha-1)} \sum_{s=a+1}^{t}(t-\rho(s)) q(s) y(s) .
\end{aligned}
$$

The boundary conditions imply that $c_{1}=0$ and

$$
\begin{aligned}
c_{2}= & \frac{2-\alpha}{(b-a) B(\alpha-1)} \sum_{s=a+1}^{b} q(s) y(s) \\
& +\frac{\alpha-1}{(b-a) B(\alpha-1)} \sum_{s=a+1}^{b}(b-\rho(s)) q(s) y(s) .
\end{aligned}
$$


Hence,

$$
\begin{aligned}
y(t)= & \frac{(2-\alpha)(t-a)}{(b-a) B(\alpha-1)} \sum_{s=a+1}^{b} q(s) y(s) \\
& -\frac{(\alpha-1)(t-a)}{(b-a) B(\alpha-1)} \sum_{s=a+1}^{b}(b-\rho(s)) q(s) y(s) \\
& -\frac{2-\alpha}{B(\alpha-1)} \sum_{s=a+1}^{t} q(s) y(s) \\
& -\frac{\alpha-1}{B(\alpha-1)} \sum_{s=a+1}^{t}(t-\rho(s)) q(s) y(s) .
\end{aligned}
$$

\section{Existence and Uniqueness Theorems for the Initial Value Problem Types}

In this section we prove existence and uniqueness theorems for CFC and CFR type initial value problems.

Theorem 14. Consider the system

$$
\begin{aligned}
& \left({ }_{a}^{C F C} \nabla^{\alpha} y\right)(t)=f(t, y(t)), \\
& t \in \mathbb{N}_{a, b}, 0<\alpha \leq 1, y(a)=c,
\end{aligned}
$$

such that $f(a, y(a))=0, A((1-\alpha) / B(\alpha)+\alpha(b-a) / B(\alpha))<1$, and $\left|f\left(t, y_{1}\right)-f\left(t, y_{2}\right)\right| \leq A\left|y_{1}-y_{2}\right|, A>0$, where $f: \mathbb{N}_{a, b} \times$ $\mathbb{R} \rightarrow \mathbb{R}$ and $y: \mathbb{N}_{a, b} \rightarrow \mathbb{R}$. Then, system (34) has a unique solution of the form

$$
y(t)=c+{ }_{a}^{C F} \nabla^{-\alpha} f(t, y(t)) .
$$

Proof. First, by the help of Proposition 10, (12), and taking into account the fact that $f(a, y(a))=0$, it is straight forward to prove that $y(t)$ satisfies system (34) if and only if it satisfies (35).

Let $X=\left\{x: \max _{t \in \mathbb{N}_{a b}}|x(t)|<\infty\right\}$ be the Banach space endowed with the norm $\|x\|=\max _{t \in \mathbb{N}_{a, b}}|x(t)|$. On $X$ define the linear operator

$$
(T x)(t)=c+{ }_{a}^{\mathrm{CF}} \nabla^{-\alpha} f(t, x(t)) .
$$

Then, for arbitrary $x_{1}, x_{2} \in X$ and $t \in \mathbb{N}_{a, b}$, we have by assumption that

$$
\begin{aligned}
& \left|\left(T x_{1}\right)(t)-\left(T x_{2}\right)(t)\right| \\
& \quad=\left|{ }_{a}^{\mathrm{CF}} \nabla^{-\alpha}\left[f\left(t, x_{1}(t)\right)-f\left(t, x_{2}(t)\right)\right]\right| \\
& \leq A\left(\frac{1-\alpha}{B(\alpha)}+\frac{\alpha(b-a)}{B(\alpha)}\right)\left\|x_{1}-x_{2}\right\|,
\end{aligned}
$$

and hence $T$ is a contraction. By Banach fixed point theorem, there exists a unique $x \in X$ such that $T x=x$ and hence the proof is complete.

Remark 15. Similar existence and uniqueness theorems can be proved for system (34) with higher order by making use of
Proposition 10. The condition $f(a, y(a))=0$ always can not be avoided as we have seen in Example 12 with $f(t, y(t))=$ $F(t)$. As a result of Theorem 14, we conclude that the fractional difference linear initial value problem

$$
\begin{aligned}
\left({ }_{a}^{\mathrm{CFC}} \nabla^{\alpha} y\right)(t) & =r y(t), \\
& r \in \mathbb{R}, t \in \mathbb{N}_{a, b}, 0<\alpha \leq 1, y(a)=c,
\end{aligned}
$$

can have only the trivial solution unless $\alpha=1$. Indeed, the solution satisfies $y(t)=c+r((1-\alpha) / B(\alpha)) y(t)+$ $(\alpha r / B(\alpha)) \sum_{s=a+1}^{t} y(s)$. This solution is only verified at $a$ if $(1-\alpha) y(a)=0$.

Theorem 16. Consider the system

$$
\begin{aligned}
& \quad\left({ }_{a}^{C F R} \nabla^{\alpha} y\right)(t)=f(t, y(t)), \\
& \quad t \in \mathbb{N}_{a, b}, \quad 1<\alpha \leq 2, y(a)=c,
\end{aligned}
$$

such that $(A / B(\alpha-1))\left((2-\alpha)(b-a)+(\alpha-1)(b-a)^{\overline{2}} / 2\right)<1$, and $\left|f\left(t, y_{1}\right)-f\left(t, y_{2}\right)\right| \leq A\left|y_{1}-y_{2}\right|, A>0$, where $f: \mathbb{N}_{a, b} \times \mathbb{R} \rightarrow \mathbb{R}$ and $y: \mathbb{N}_{a, b} \rightarrow \mathbb{R}$. Then, system (34) has a unique solution of the form

$$
\begin{aligned}
y(t)= & c+{ }_{a}^{C F} \nabla^{-\alpha} f(t, y(t)) \\
= & c+\frac{2-\alpha}{B(\alpha-1)} \sum_{s=a+1}^{t} f(s, y(s)) \\
& +\frac{\alpha-1}{B(\alpha-1)}\left({ }_{a} \nabla^{-2} f(\cdot, y(\cdot))(t)\right) .
\end{aligned}
$$

Proof. If we apply ${ }_{a}^{\mathrm{CF}} \nabla^{-\alpha}$ to system (39) and make use of Proposition 10 with $\beta=\alpha-1$ then we reach at the representation (40). Conversely, if we apply ${ }_{a}^{\mathrm{CFR}} \nabla^{\alpha}$, make use of Proposition 10 and by noting that

$$
{ }_{a}^{\mathrm{CFR}} \nabla^{\alpha}={ }_{a}^{\mathrm{CFR}} \nabla^{\beta} \nabla_{t} c=0,
$$

we obtain system (39). Hence, $y(t)$ satisfies system (39) if and only if it satisfies (40).

Let $X=\left\{x: \max _{t \in \mathbb{N}_{a, b}}|x(t)|<\infty\right\}$ be the Banach space endowed with the norm $\|x\|=\max _{t \in \mathbb{N}_{a, b}}|x(t)|$. On $X$ define the linear operator

$$
(T x)(t)=c+{ }_{a}^{\mathrm{CF}} \nabla^{-\alpha} f(t, x(t)) .
$$

Then, for arbitrary $x_{1}, x_{2} \in X$ and $t \in \mathbb{N}_{a, b}$, we have by assumption that

$$
\begin{aligned}
& \left|\left(T x_{1}\right)(t)-\left(T x_{2}\right)(t)\right| \\
& \quad=\left|{ }_{a}^{\mathrm{CF}} \nabla^{-\alpha}\left[f\left(t, x_{1}(t)\right)-f\left(t, x_{2}(t)\right)\right]\right| \\
& \quad \leq \frac{A}{B(\alpha-1)}\left((2-\alpha)(b-a)+\frac{(\alpha-1)(b-a)^{\overline{2}}}{2}\right) \\
& \cdot\left\|x_{1}-x_{2}\right\|,
\end{aligned}
$$

and hence $T$ is a contraction. By Banach Contraction Principle, there exists a unique $x \in X$ such that $T x=x$ and hence the proof is complete. 


\section{The Lyapunov Inequality for the CFR Difference Boundary Value Problem}

In this section, we prove a Lyapunov inequality for a CFR boundary value difference problem of order $2<\alpha \leq 3$.

Consider the boundary value problem

$$
\begin{aligned}
& \left({ }_{a}^{\mathrm{CFR}} \nabla^{\alpha} y\right)(t)+q(t) y^{\rho}(t)=0, \\
& 2<\alpha \leq 3, t \in \mathbb{N}_{a+1, b-1}, y(a)=y(b)=0,
\end{aligned}
$$

where, $y^{\rho}(t)=y(\rho(t))=y(t-1)$.

Lemma 17. $y(t)$ is a solution of the boundary value problem (44) if and only if it satisfies the equation

$$
y(t)=\sum_{s=a+1}^{b} G(t, s) T(s, y(s)),
$$

where

$$
\begin{aligned}
& G(t, s) \\
& = \begin{cases}\frac{(t-a)(b-\rho(s))}{b-a}, & t+1 \leq s, t, s \in \mathbb{N}_{a, b}, \\
\left(\frac{(t-a)(b-\rho(s))}{b-a}-(t-\rho(s))\right), & s-1 \leq t, t, s \in \mathbb{N}_{a, b},\end{cases} \\
& T(t, y(t))={ }_{a}^{C F} \nabla^{\beta}\left(q(\cdot) y^{\rho}(\cdot)\right)(t) \\
& =\frac{1-\beta}{B(\beta)} q(t) y^{\rho}(t)+\frac{\beta}{B(\beta)}\left({ }_{a} \nabla^{-1} q(\cdot) y^{\rho}(\cdot)\right)(t), \quad \beta=\alpha-2 .
\end{aligned}
$$

Proof. Apply the integral ${ }_{a}^{\mathrm{CF}} \nabla^{-\alpha}$ to (44) and make use of Definition 6 and Proposition 10 with $n=2$ and $\beta=\alpha-2$ to reach

$$
\begin{aligned}
y(t) & =c_{1}+c_{2}(t-a)-\left({ }_{a} \nabla^{-2} T(\cdot, y(\cdot))\right)(t) \\
& =c_{1}+c_{2}(t-a)-\sum_{s=a+1}^{t}(t-\rho(s)) T(s, y(s)) .
\end{aligned}
$$

The condition $y(a)=0$ implies that $c_{1}=0$ and the condition $y(b)=0$ implies that $c_{2}=(1 /(b-a)) \sum_{s=a+1}^{b}(b-$ $\rho(s)) T(s, y(s))$, and hence

$$
\begin{aligned}
y(t)= & \frac{t-a}{b-a} \sum_{s=a+1}^{b}(b-\rho(s)) T(s, y(s)) \\
& -\sum_{s=a+1}^{t}(t-\rho(s)) q(s) T(s, y(s)) d s .
\end{aligned}
$$

Then, the result follows by splitting the summation

$$
\begin{aligned}
\sum_{s=a+1}^{b} & (b-\rho(s)) T(s, y(s)) \\
= & \sum_{s=a+1}^{t}(b-\rho(s)) T(s, y(s)) \\
& +\sum_{s=t+1}^{b}(b-\rho(s)) T(s, y(s)) .
\end{aligned}
$$

Lemma 18. Given that $b \equiv a(\bmod 1)$, the Green function $G(t, s)$ defined in Lemma 17 has the following properties:

(1) $G(t, s) \geq 0$ for all $t, s \in \mathbb{N}_{a, b}$.

(2) $\max _{t \in \mathbb{N}_{a, b}} G(t, s)=G(\rho(s), s)$ for $s \in \mathbb{N}_{a+1, b-1}$.

(3) $f(s)=G(\rho(s), s)=(\rho(s)-a)(b-\rho(s)) /(b-a)$ has $a$ unique maximum, given by

$$
\begin{aligned}
& \max _{s \in \mathbb{N}_{a, b} f(s)} \\
& = \begin{cases}f\left(\frac{(a+b+2)}{2}\right)=\frac{b-a}{4} & \text { if } b-a \text { is even } \\
f\left(\frac{(a+b+3)}{2}\right)=\frac{(b-a)^{2}-1}{4(b-a)} & \text { if } b-a \text { is odd. }\end{cases}
\end{aligned}
$$

Hence, in either cases $\max _{s \in \mathbb{N}_{a, b}} f(s) \leq(b-a) / 4$.

Proof. (1) It is clear that $g_{1}(t, s)=(t-a)(b-\rho(s)) /(b-a) \geq 0$. Regarding the part $g_{2}(t, s)=((t-a)(b-\rho(s)) /(b-a)-(t-\rho(s)))$ we see that $(t-\rho(s))=((t-a) /(b-a))(b-(a+(\rho(s)-a)(b-$ $a) /(t-a)))$ and that $a+(\rho(s)-a)(b-a) /(t-a) \geq \rho(s)$ if and only if $\rho(s)(b-t)+a(t-b) \geq 0$ if and only if $s \geq a+1$. Hence, we conclude that $g_{2}(t, s) \geq 0$ as well.

(2) Clearly, $g_{1}(t, s)$ is an increasing function in $t$. Applying $\nabla$ to $g_{2}$ with respect to $t$ for every fixed $s \geq a+1$ we see that $\nabla_{t} g(t, s)=(b-a)^{-1}(a-\rho(s))$ and hence $g_{2}$ is a decreasing function in $t$. Then,

(3) Let $f(s)=G(\rho(s), s)=(\rho(s)-a)(b-\rho(s)) /(b-a)$.

$$
(\nabla f)(s)=\frac{a+b-2 s+3}{b-a}=0,
$$

if $s=(a+b+3) / 2$ and hence for $a, b \in \mathbb{N}, f$ attains its maximum at $s_{1}=(a+b+3) / 2$ if $a+b($ or $b-a)$ is odd and at $(a+b+2) / 2$ if $a+b$ (or $b-a)$ is even. More generally, if $a$ and $b$ are such that $b \equiv a(\bmod 1)$, we see that $s_{1} \equiv a(\bmod 1)$ if $b-a$ is odd and $s_{2} \equiv a(\bmod 1)$ if $b-a$ is even. Finally, $f\left(s_{1}\right)=\left((b-a)^{2}-1\right) / 4(b-a)$ and $f\left(s_{2}\right)=(b-a) / 4$.

In next lemma, we estimate $T(t, y(t))$ for a function $y \in B\left[\mathbb{N}_{a, b}\right]$, the Banach space of all Banach-valued finite sequences on $\mathbb{N}_{a, b}$ with $\|y\|=\max _{t \in \mathbb{N}_{a, b}}|y(t)|$, where $|\cdot|$ is the norm in the Banach space.

Lemma 19. For $y \in B\left[\mathbb{N}_{a, b}\right]$ and $2<\alpha \leq 3, \beta=\alpha-2$, we have, for any $t \in \mathbb{N}_{a, b}$,

$$
|T(t, y(t))| \leq R(t)\|y\|,
$$

where

$$
R(t)=\left[\frac{3-\alpha}{B(\alpha-2)}|q(t)|+\frac{\alpha-2}{B(\alpha-2)} \sum_{s=a+1}^{t}|q(s)|\right] .
$$

Theorem 20 (the CFR fractional difference Lyapunov inequality). If the boundary value problem (44) has a nontrivial solution, where $q(t)$ is a real-valued bounded function on $\mathbb{N}_{a, b}$, then

$$
\sum_{s=a+1}^{b} R(s)>\frac{4}{b-a} .
$$


Proof. Assume $y \in Y=B\left[\mathbb{N}_{a, b}\right]$ is a nontrivial solution of the boundary value problem (44). By Lemma 17, $y$ must satisfy

$$
y(t)=\sum_{s=a+1}^{b} G(t, s) T(s, y(s)) .
$$

Then, by using the properties of the Green function $G(t, s)$ proved in Lemmas 18 and 19, we come to the conclusion that

$$
\|y\|<\frac{b-a}{4} \sum_{s=a+1}^{b} R(s)\|y\|
$$

from which (54) follows.

Remark 21. Note that if $\alpha \rightarrow 2^{+}$, then $R(t)$ tends to $|q(t)|$ and hence we obtain the classical nabla discrete version of the Lyapunov inequality (2). For the sake of more comparisons of Lyapunov inequalities on time scales we refer to [40].

Example 22. Consider the following CFR Sturm-Liouville difference eigenvalue problem (SLDEP) of order $2<\alpha \leq 3$

$$
\begin{aligned}
& \left({ }_{0}^{\mathrm{CFR}} \nabla^{\alpha} y\right)(t)+\lambda y^{\rho}(t)=0, \\
& \quad t \in \mathbb{N}_{1, b-1}, \quad b \in \mathbb{N}, \quad y(0)=y(b)=0 .
\end{aligned}
$$

If $\lambda$ is an eigenvalue of (57), then by Theorem 20 with $q(t)=\lambda$, we have

$$
\begin{aligned}
T(t) & =\left[\frac{3-\alpha}{B(\alpha-2)}|\lambda|+\frac{\alpha-2}{B(\alpha-2)}\left({ }_{0} \nabla^{-1}|\lambda|\right)(t)\right] \\
& =|\lambda|\left[\frac{3-\alpha}{B(\alpha-2)}+\frac{\alpha-2}{B(\alpha-2)} t\right] .
\end{aligned}
$$

Hence, we must have

$$
\sum_{s=1}^{b} T(s)=|\lambda|\left[\frac{b(3-\alpha)}{B(\alpha-2)}+\frac{b^{\overline{2}}(\alpha-2)}{2 B(\alpha-2)}\right]>\frac{4}{b} .
$$

Notice that the limiting case $\alpha \rightarrow 2^{+}$implies that $|\lambda|>4 / b^{2}$ which is the lower bound for the eigenvalues of the ordinary difference eigenvalue problem:

$$
\begin{aligned}
& \left(\nabla^{2} y\right)(t)+\lambda y^{\rho}(t)=0, \\
& \qquad t \in \mathbb{N}_{1, b-1}, y(0)=y(b)=0 .
\end{aligned}
$$

\section{Conclusions}

Fractional differences and their correspondent fractional sum operators are of importance in discrete modeling of various problems in science. We extended the fractional difference calculus whose difference operators depend on a discrete exponential function kernel to arbitrary positive order. The correspondent arbitrary order fractional sum operators have been defined as well and applied to solve fractional initial and boundary value difference problems. The extension for right fractional differences and sums is also achieved. To set up the basic concepts, we proved existence and uniqueness theorems by means of Banach fixed point theorem for initial value problems in the frame of CFC and CFR fractional differences. We have come to the conclusion that the condition $f(a, y(a))=0$ is necessary to guarantee the existence of solution and hence fractional linear difference initial value problem with constant coefficients results in the trivial solution unless the order is positive integer. We used our extension to arbitrary order to prove a Lyapunov type inequality for a CFR boundary value problem of order $2<$ $\alpha \leq 3$ and then obtain the classical ordinary case when $\alpha$ tends to 2 from right. This proves different behavior from the classical fractional difference case, where the Lyapunov inequality was proved for a fractional difference boundary problem of order $1<\alpha \leq 2$ and the classical ordinary case was then recovered when $\alpha$ tends to 2 from left.

\section{Conflicts of Interest}

The authors declare that they have no conflicts of interest.

\section{Acknowledgments}

The first author would like to thank the Research and Translation Center in Prince Sultan University for continuous encouragement and support.

\section{References}

[1] M. A. Hajji, Q. M. Al-Mdallal, and F. M. Allan, "An efficient algorithm for solving higher-order fractional Sturm-Liouville eigenvalue problems," Journal of Computational Physics, vol. 272 , pp. 550-558, 2014.

[2] Q. M. Al-Mdallal and M. A. Hajji, "A convergent algorithm for solving higher-order nonlinear fractional boundary value problems," Fractional Calculus and Applied Analysis, vol. 18, no. 6, pp. 1423-1440, 2015.

[3] Q. M. Al-Mdallal, "On the numerical solution of fractional Sturm-Liouville problems," International Journal of Computer Mathematics, vol. 87, no. 12, pp. 2837-2845, 2010.

[4] A. H. Bhrawy, T. M. Taha, and J. A. Machado, "A review of operational matrices and spectral techniques for fractional calculus," Nonlinear Dynamics. An International Journal of Nonlinear Dynamics and Chaos in Engineering Systems, vol. 81, no. 3, pp. 1023-1052, 2015.

[5] F. Wu and J. Liu, "Discrete fractional creep model of salt rock," J. Comput. Complex. Appl, vol. 2, pp. 1-6, 2016.

[6] R. Abu-Saris and Q. Al-Mdallal, "On the asymptotic stability of linear system of fractional-order difference equations," Fractional Calculus and Applied Analysis. An International Journal for Theory and Applications, vol. 16, no. 3, pp. 613-629, 2013.

[7] T. Abdeljawad and D. Baleanu, "On fractional derivatives with exponential kernel and their discrete versions," Journal of Reports in Mathematical Physics, https://arxiv.org/abs/1606 .07958 .

[8] M. Caputo and M. Fabrizio, "A new definition of fractional derivative without singular kernal," Progress in Fractional Differentiation and Applications, vol. 1, no. 2, pp. 73-85, 2015. 
[9] J. Losada and J. J. Nieto, "Properties of a new fractional derivative without singular kernal," Progress in Fractional Differentiation and Applications, vol. 1, no. 2, pp. 87-92, 2015.

[10] T. Abdeljawad and D. Baleanu, "Monotonicity results for fractional difference operators with discrete exponential kernels," Advances in Difference Equations, vol. 2017, Article ID 78, 2017.

[11] A. Atangana and D. Baleanu, "New fractional derivatives with non-local and non-singular kernel: Theory and application to heat transfer model," Thermal Science, vol. 20, no. 2, pp. $763-$ 769, 2016.

[12] T. Abdeljawad and D. Baleanu, "Integration by parts and its applications of a new nonlocal fractional derivative with MittagLeffler nonsingular kernel," The Journal of Nonlinear Sciences and Applications, vol. 10, no. 03, pp. 1098-1107, 2017.

[13] T. Abdeljawad and D. Baleanu, "Discrete fractional differences with nonsingular discrete Mittag-Leffler kernels," Advances in Difference Equations, vol. 2016, Article ID 232, 2016.

[14] T. Abdeljawad and D. Baleanu, "Monotonicity analysis of a nabla discrete fractional operator with discrete Mittag-Leffler kernel," Chaos, Solitons \& Fractals, 2017.

[15] R. A. Ferreira, "Some discrete fractional Lyapunov-type inequalities," Fractional Differential Calculus, vol. 5, no. 1, pp. 87-92, 2015.

[16] R. A. Ferreira, "A Lyapunov-type inequality for a fractional boundary value problem," Fractional Calculus and Applied Analysis. An International Journal for Theory and Applications, vol. 16, no. 4, pp. 978-984, 2013.

[17] A. Chidouh and D. F. Torres, "A generalized Lyapunov's inequality for a fractional boundary value problem," Journal of Computational and Applied Mathematics, vol. 312, pp. 192-197, 2017.

[18] M. Jleli and B. Samet, "Lyapunov-type inequalities for fractional boundary-value problems," Electronic Journal of Differential Equations, vol. 2015, no. 88, 11 pages, 2015.

[19] D. O'Regan and B. Samet, "Lyapunov-type inequalities for a class of fractional differential equations," Journal of Inequalities and Applications, vol. 2015, no. 247, 10 pages, 2015.

[20] J. Rong and C. Bai, "Lyapunov-type inequality for a fractional differential equation with fractional boundary conditions," Advances in Difference Equations, vol. 2015, no. 82, 10 pages, 2015.

[21] M. Jleli and B. Samet, "A Lyapunov-type inequality for a fractional $q$-difference boundary value problem," Journal of Nonlinear Science and its Applications. JNSA, vol. 9, no. 5, pp. 1965-1976, 2016.

[22] H. Esser, "Stability inequalities for discrete nonlinear two-point boundary value problems," Applicable Analysis. An International Journal, vol. 10, no. 2, pp. 137-162, 1980.

[23] N. G. Abuj and D. B. Pachpatte, "Lyapunov type inequality for fractional differential equation with k-Prabhakar derivative," https://arxiv.org/abs/1702.01562.

[24] D. B. Pachpatte, N. G. Abuj, and A. D. Khandagale, "Lyapunov type inequality for hybrid fractional differential equation with Prabhakar Derivative," https://arxiv.org/abs/1609.03027.

[25] X. Yang, "On Liapunov-type inequality for certain higher-order differential equations," Applied Mathematics and Computation, vol. 134, no. 2-3, pp. 307-317, 2003.

[26] A. n. Tiryaki, "Recent developments of Lyapunov-type inequalities," Advances in Dynamical Systems and Applications, vol. 5, no. 2, pp. 231-248, 2010.
[27] N. Parhi and S. Panigrahi, "Liapunov-type inequality for higher order differential equations," Mathematica Slovaca, vol. 52, no. 1, pp. 31-46, 2002.

[28] J. X. Cui, X. Huang, and M. C. Hou, "Lyapunov-type inequality for fractional order difference equations," Global Journal of Science Frontier Research, vol. 16, no. 1, 2016.

[29] T. Abdeljawad, "A Lyapunov type inequality for fractional operators with nonsingular Mittag-Leffler kernel," Journal of Inequalities and Applications, 2017:130 pages, 2017.

[30] A. M. Lyapunov, "Probleme général de la stabilité du mouvment," Annales de la Faculté des Sciences de Toulouse, vol. 2, pp. 227-247, 1907, Reprinted in: Ann. Math. Studies, no. 17, Princeton, 1947.

[31] S. S. Cheng, "A discrete analogue of the inequality of Lyapunov," Hokkaido Mathematical Journal, vol. 12, no. 1, pp. 105-112, 1983.

[32] S. G. Samko, A. A. Kilbas, and O. I. Marichev, Fractional Integrals and Derivatives, Theory and Applications, Gordon and Breach, Yverdon, Switzerland, 1993.

[33] I. Podlubny, Fractional Differential Equations, vol. 198 of Mathematics in Science and Engineering, Academic Press, San Diego, Calif, USA, 1999.

[34] A. Kilbas, M. H. Srivastava, and J. J. Trujillo, Theory and Application of Fractional Differential Equations, vol. 204 of North Holland Mathematics Studies, 2006.

[35] F. Bozkurt, T. Abdeljawad, and M. A. Hajji, "Stability analysis of a fractional order differential equatin model of a brain tumor growth depending on the density," Applied and Computational Mathematics. An International Journal, vol. 14, no. 1, pp. 50-62, 2015.

[36] C. Goodrich and A. C. Peterson, Discrete Fractional Calculus, Springer International, New York, NY, USA, 2015.

[37] T. Abdeljawad, "On delta and nabla Caputo fractional differences and dual identities," Discrete Dynamics in Nature and Society, vol. 2013, Article ID 406910, 12 pages, 2013.

[38] T. Abdeljawad, "Dual identities in fractional difference calculus within Riemann," Advances in Difference Equations, vol. 2013, no. 36, 2013.

[39] T. Abdeljawad and D. F. M. Torres, "Symmetric duality for left and right Riemann-Liouville and Caputo fractional differences," Arab Journal of Mathematical Sciences, vol. 23, no. 2, pp. 157-172, 2017.

[40] M. Bohner and A. Peterson, Dynamic equations on time scales, Springer, Berlin, Germany, 2001. 


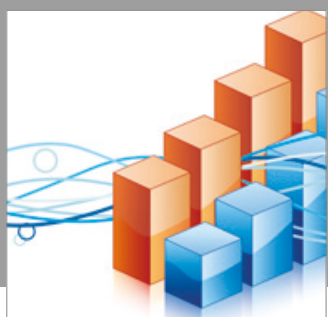

Advances in

Operations Research

vatersals

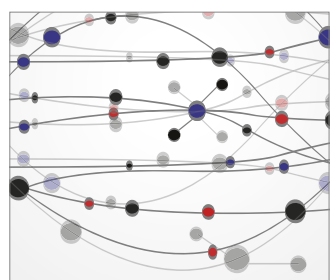

\section{The Scientific} World Journal
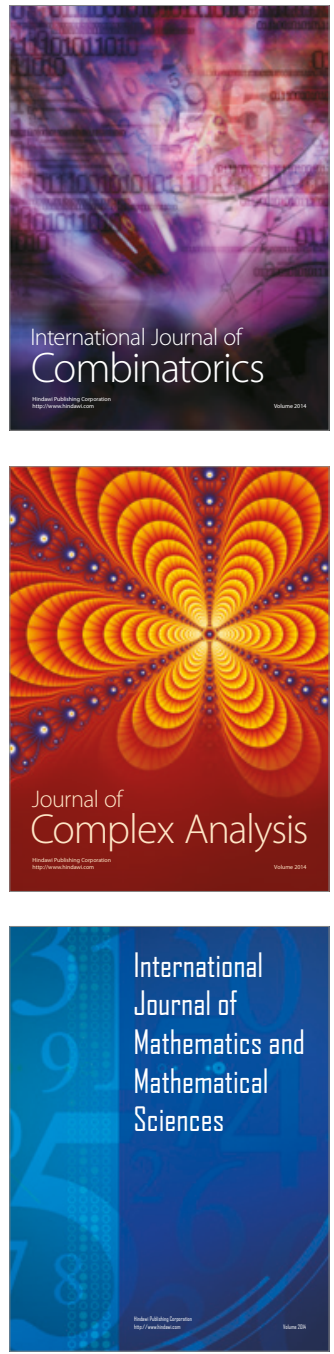
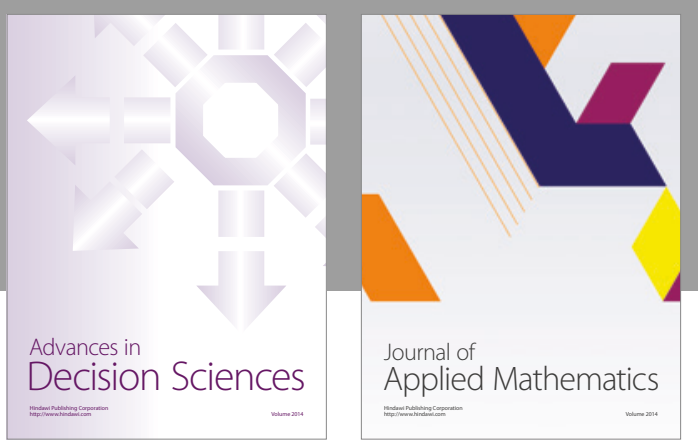

Algebra

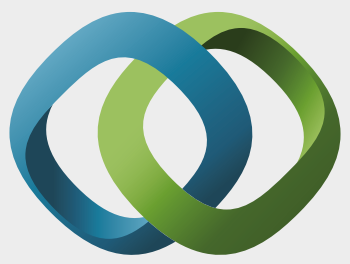

\section{Hindawi}

Submit your manuscripts at

https://www.hindawi.com
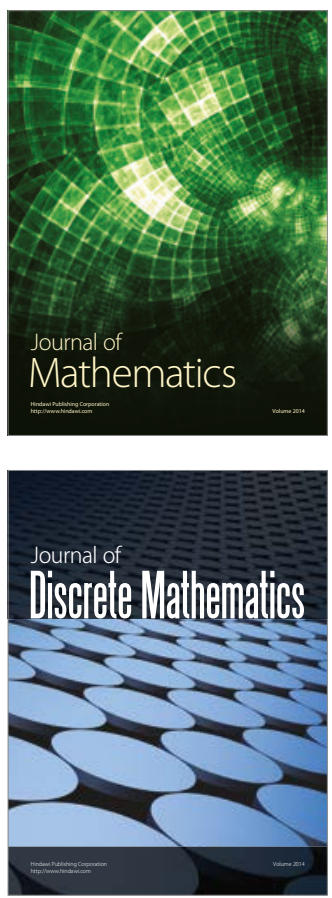

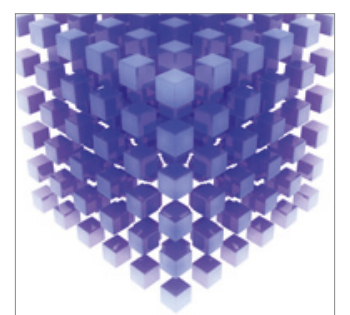

Mathematical Problems in Engineering
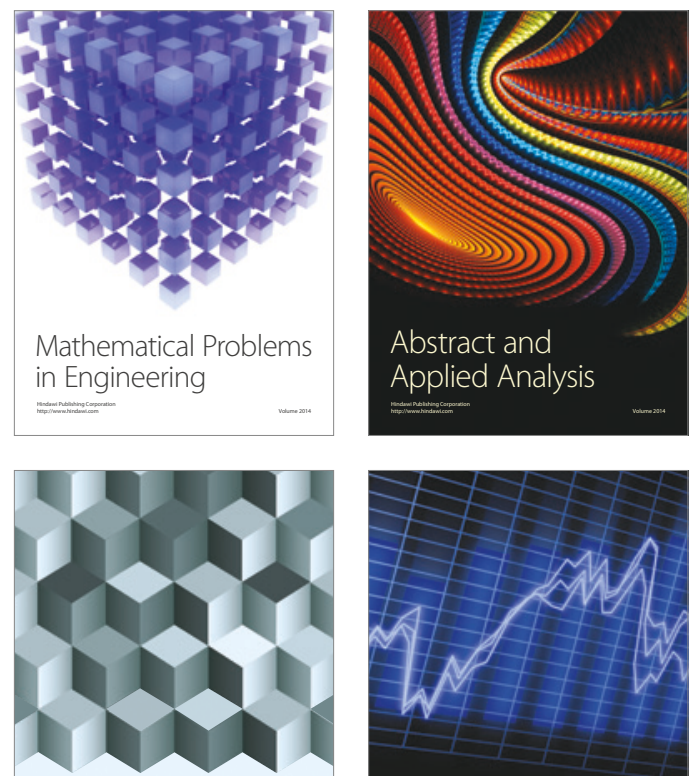

Journal of

Function Spaces

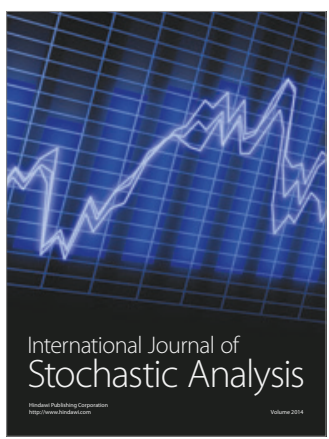

Probability and Statistics
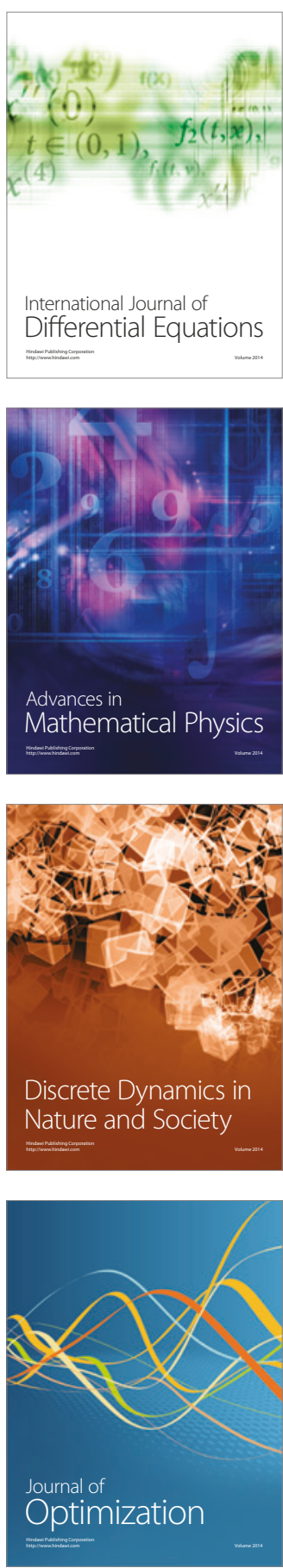